PACS: 61.80.-x, 07.89.+b

\title{
Radiation effects on electronic circuits in a spatial environment
}

\author{
N. Merabtine ${ }^{1}$, M. Benslama ${ }^{1}$, A. Benslama², Dj. Sadaoui ${ }^{3}$ \\ 1 Laboratoire électromagnétisme et Télécommunications, département d'électronique faculté des sciences de \\ l'ingénieur Université Mentouri Constantine Algeria \\ 2 Laboratoire de Physique atomique et Subatomique, département de physique faculté des sciences université \\ Mentouri Constantine Algeria \\ 3 Département d'électronique université de Jijel Algeria \\ E-mail:na_merabtine@hotmail.com
}

\begin{abstract}
The failings bound to the deterioration of the components under irradiations are complex and numerous. This paper describes the different kinds of radiations present in the space as well as their interactions with the materials composing the integrated circuits used in the spacecraft. Our survey will be focused on the Single Event Upset phenomenon induced by the passage of only one ionizing particle with applications on the Samsung SDRAM.
\end{abstract}

Keywords: spatial environment, ionizing radiations, heavy ions, protons, SEU (Single Event Upset).

Manuscript received 11.09.04; accepted for publication 16.12.04.

\section{Introduction}

Historically, thirty years ago, one observed on the electronic circuits embarked on board of spacecraft the first malfunctions caused by the radiations of the space. With the considerable expansion of the spatial activity in the eighties new electronic dysfunction appeared which are bound to radiances. Next to the dose phenomenon, that comes progressively, but in a permanent way altering the circuits working, singular events induced by the passage of an unique ionizing particle occur frequently (SEE: Single Event Effect). Among the transient risks, one can quote: the upsets (SEU: Single Event Upset, Logical toppling over of one memory point) and the latch-up (SEL: Single Event Latch-up).

In the space the upsets are mainly caused by the heavy ions and the protons. The role of the energising protons of a few hundred of $\mathrm{MeV}$ had been neglected for a long time because they don't induce sufficient energy deposits to provoke directly errors. However the increase of the error rates in the circuits during the solar eruption or during the passage of Satellites inside the belts of radiations has underlined the risks they represent.

\section{The radiative spatial environment}

In orbit all spatial vehicles are submitted to a set of radiative contraints that come from the sun, the galactic space and extragalactic [1]. The Fig. 1 and Table summarize the source and the nature of the particles met in the space as well as their energies in the magnetosphere. It permits to have an overall view of the ionizing radiances likely to meet the components at the time of spatial mission.

\subsection{The cosmic radiances}

The "cosmic radiances" refer to the set of big intensity radiances with strong power of penetration that propagate in the interstellar space.

Discovered by V. Hess in 1912, the nature of this radiance has not been maintained until 1950. This latter is constituted of about $1 \%$ of ions (nucleus of heavy atoms deprived of a part of their electronic procession) of very big energy $(E>1 \mathrm{MeV}), 85 \%$ of protons and $14 \%$ of Helium nucleis $[3,4]$.

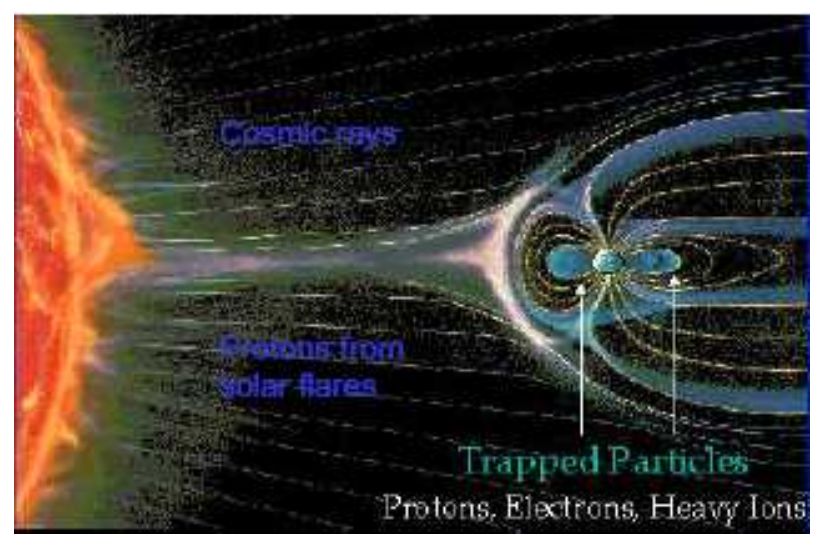

Fig. 1. The charged particles come from three sources: cosmic rays, van Allen belts and solar flare [2]. 
These energizing particles constituting the radiance would be produced at the time of supernovae (old star explosion) [5], then interact with the clouds of interstellar gas and are deviated along the force lines of galactic magnetic field.

\subsection{The radiations belts}

The radiations belts or van Allen belts are a part of the earth magnetosphere structure. They were discovered at the end of fifties during the launching of the first Russian and American artificial satellites. These belts are essentially constituted of electrons and ions (protons mainly) very energizing whose movement around the earth is strongly constrained by the earth magnetic field. They are notably at the origin of the deterioration of some satellite components (such as the solar panels), parasitic signal in the embarked electronic devices and the electric discharge outbreak between different parts of the satellite. We must note, without taking into account the magnetospherical instabilities, that the loaded particles fluxes met by a satellite in orbit vary according to the altitude and the inclination; especially in low orbit the radiation belt being close to the earth at high latitudes as well as in the anomaly of the South Atlantic where the fluxes can be neglected for all orbit portions situated outside these zones.

A satellite in equatorial low orbit is thus not pratically exposed to radiations. For a slant not exceeding $45^{\circ}$, the only particles met are the protons of the south atlantic anomaly.

The van Allen belts are distributed on two zones around the Earth:

- an inner belt, starting between 300 and $1000 \mathrm{~km}$ altitude (according to the latitude) and spreading until $10000 \mathrm{~km}$ altitude, populated mainly by very energizing protons, several hundreds of millions of electronvolts $(\mathrm{MeV})[6]$;

- an outer belt, starting at $10,000 \mathrm{~km}$ altitude and spreading beyond the geostationnary orbits $(36,000 \mathrm{~km}$ altitude) and populated mainly by electrons of a few $\mathrm{MeV}$ [6].

\subsection{The particles of solar origin}

\subsubsection{The solar eruptions}

The solar eruptions discovered by Galilleo in 1610. The solar stains, visible manifestation of the disruptions of the matter movements and magnetic fields at the sun surface, can evolve to an eruptive event during which enormous energy quantities are liberated as electromagnetic radiance at very large spectrum (from gamma rays to radio waves). It appears, counting the number of yearly stains, a cyclic solar activity whose periodicity is the order of 11 years, itself modulated by a cycle of 80 years. In these solar cycles of 11 years, we rudely distinguish 4 years of weak activity and 7 years of strong activity punctuated by minor and exceptional events.

In fact, it is necessary to distinguish, in the frame of the only radiatiance mentioned here, two solar eruption types:

- the protons solar eruptions, whose life duration varies from a few hours to a few days, and whose main emission is constituted of protons of important energies (until a few hundreds of $\mathrm{MeV}$ );

- the heavy ions solar eruptions, whose main emission is constituted of heavy ions (of a variable composition from an eruption to another).

The protons and heavy ions which can be emitted during these eruptions appear as very dangerous by the deteriorations they are likely to provoke in the embarked systems [7].

\subsubsection{The solar wind}

The interplanetary environment is permanently swept by a wind of electrically charged particles from the sun, this wind is very little dense ( 10 particles by $\mathrm{cm}^{3}$ ) but very fast (400 to $800 \mathrm{~km} / \mathrm{s}$ ), it is a supersonic wind (it moves quicker than the sonor waves) [8].

The solar wind is mainly composed of protons and electrons of low energy (a few keV). These particles are not likely to induce some disruptions in the embarked components, they can however be a cause of the electronic systems working anomalies, when the

Synthetic presentation of the radiative spatial environment.

\begin{tabular}{|l|l|l|}
\hline \multicolumn{1}{|c|}{ Origin } & \multicolumn{1}{c|}{ Particles } & \multicolumn{1}{c|}{ Energies } \\
\hline Radiations belts & $\begin{array}{l}\text { protons ( low Earth orbit ) } \\
\text { electrons (Geo.Earth orbit ) }\end{array}$ & $\begin{array}{l}<10 \mathrm{MeV} \\
<2 \mathrm{MeV}\end{array}$ \\
\hline Solar wind & $\begin{array}{l}\text { protons } \\
\text { electrons }\end{array}$ & $\begin{array}{l}<100 \mathrm{keV} \\
\text { a few keV }\end{array}$ \\
\hline Solar eruptions & $\begin{array}{l}10 \mathrm{MeV} \text { to } 1 \mathrm{GeV} \\
\text { protons } \\
\text { heavy ions }\end{array}$ & $\begin{array}{l}10 \mathrm{MeV} \text { to a few } 100 \mathrm{MeV} \\
10^{2} \text { to } 10^{6} \mathrm{MeV} \\
1 \mathrm{MeV} \text { to } 10^{14} \mathrm{MeV}\end{array}$ \\
\hline Cosmic rays & $\begin{array}{l}\text { protons } \\
\text { heavy ions }\end{array}$ & \\
\hline
\end{tabular}




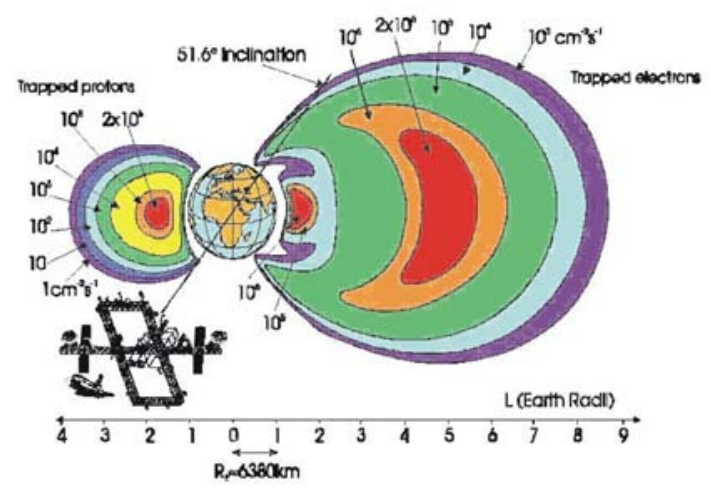

Fig. 2. Van Allen radiations belts.

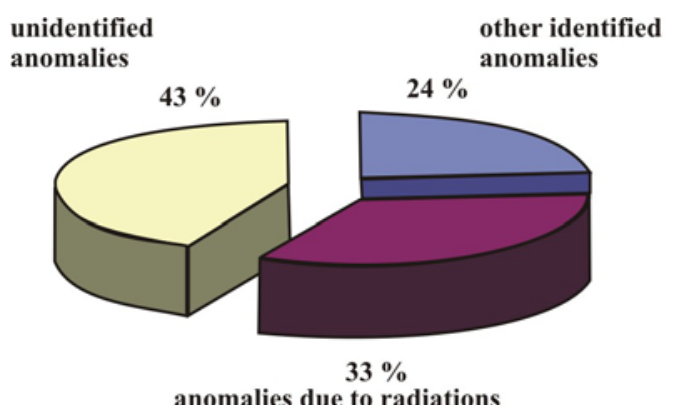

Fig. 3. Proportion of the anomalies due to the effect of radiations on board of a satellite.

electrostatic discharges occur at the level of coatings of the satellites thermal control.

\section{Radiation effects on integrated circuits}

The electronic systems embarked on board of spacecrafts (rocket, probe, satellite, shuttle) are submitted to a permanent radiance of either cosmic or solar origin. These radiations provoke physical failings of the high integration electronic components that reverbate to the electrical and logical level. This is how transitory effects named SEE can be provoked by the passage of an unique charged particle through sensitive zones of the circuits.

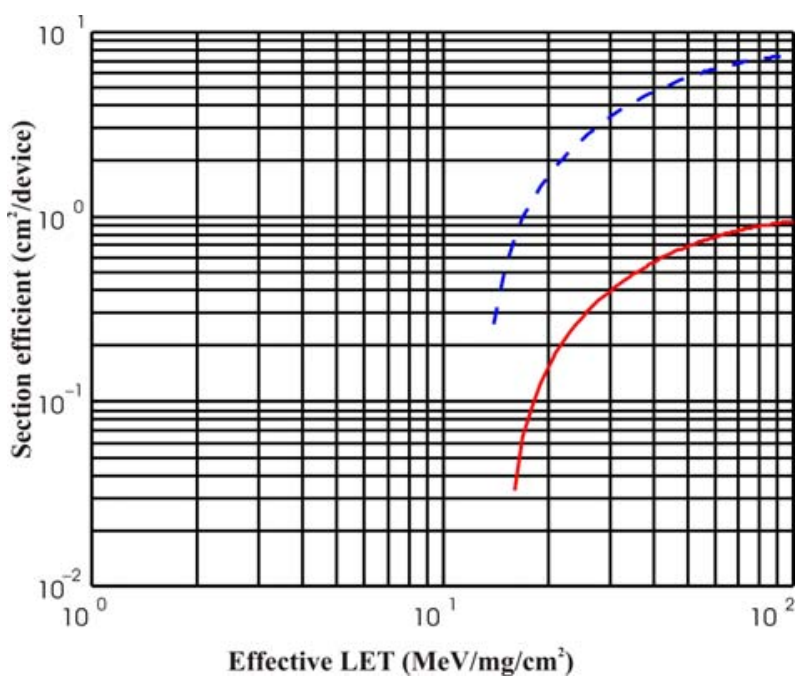

Fig. 4. Upset efficient section induced by heavy ions according to the effective LET (Samsung $64 \mathrm{Mb}$ SDRAM).

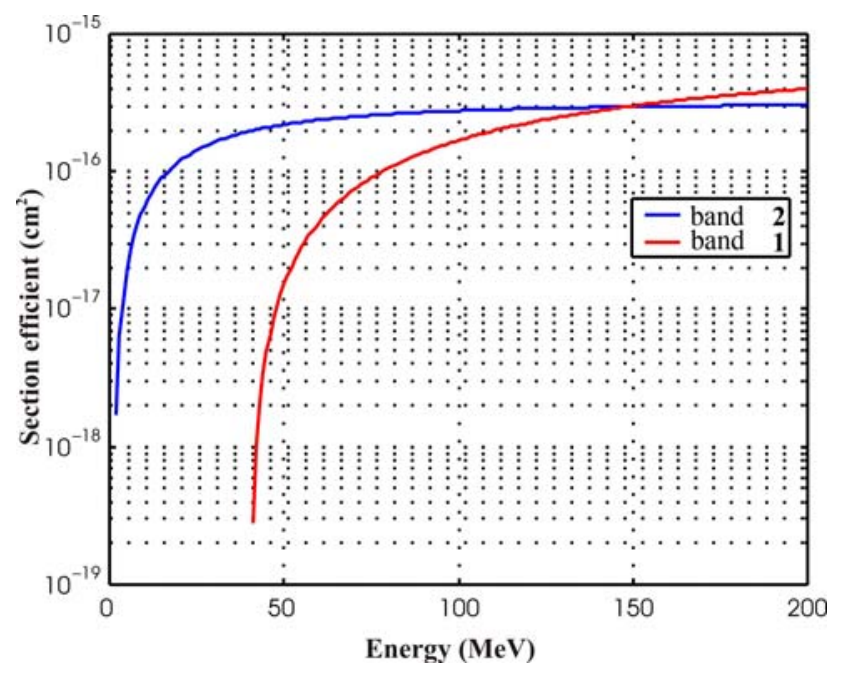

Fig. 5. Upset efficient section induced by proton according to the proton incident energy (SMART - 1 DRAM).

The natural particles interacting with the integrated circuits are essentially the photons, electrons, $\alpha$ particles and ions.

The more important are the mass and the energy of these particles, the more damages they provoke and are difficult to stop or to divert.

While penetrating in a material, a loaded particle meets a lot of emptiness and in case of contact, strickes principally electrons.

As an indicative title, the Fig. 3 gives a preview of the type and the proportion of failings occuring in a satellite [8]. 


\subsection{Dose effect}

The dose effect is defined as the energy deposited in a given material by unit of mass [9-10].

The dose effects are common to all environment.

This deposited energy is likely to modify the electrical characteristics of the component.

If we don't operate any particular treatments, the doses superior to $1 \mathrm{krad}$ are likely to generate serious problems.

\subsection{The singular events}

In the beginning of the eighties, an effect of radiations associated to heavy ions of the cosmic radiance as well as the secondaries created by nuclear reaction by the trapped protons or of solar eruption had been noted on orbit [10], the consideration of the heavy ions effects is of a major importance in the choice of some components for the satellite reliability and concerns particulary high integration circuits.

The passage of an ionizing particles (of a primary or secondary origin) in a component can generate different types of events, which may lead to failing or even to the destruction of the component.

The SEU is the change of state of a memory point. This effect is not destructive. Its study is the aim of this paper.

The SEL (Single Event Latch-up) results from the conduction of a $p-n-p-n$ parasite structure (Thyristor effect) in a CMOS component.

The latch-up creates a direct path between the mass and the power supply and consequently causes an important warming-up of the component capable of leading to its deterioration [11].

\section{Single Event Upset}

The ionization of the materials, further to the passage of a charged particle in a circuit, may cause an "Upset" phenomenon (named also "bit flip" in the English Literature) that is the inopportune change of a bistable state. The upsets effect in priority the memory points.

The incident particle produces electron-hole paires along its trajectory [5] and makes appear a current impulse which, if the amplitude and the duration are sufficient enough, can produce the same effect as the one of an outside signal applied to the transistor.

The consequences of the SEU on the behaviour of integrated circuits components depend on the nature of the information contained in the unsettled cells and the instant of occurrence and can have in certain cases critical consequences.

\subsection{SEU lead by heavy ions}

An ionizing particle, during its passage in a component, deposits the energy by ionization of the matter all along its trace.

This deposit energy by unit of length, $d E / d x$, corresponds to the creation of electron-hole paire, this dimension is called LET (Linear Energy Transfer) [12].

For a given component, the ion effect leads to a failing only above a minimal LET called Threshold LET, that is expressed in $\mathrm{MeV} \cdot \mathrm{cm}^{2} \cdot \mathrm{mg}^{-1}$ or in $\mathrm{pC} / \mu \mathrm{m}$. For a flux of ions of a given LET the events number then accounted are as important as the sensitive surface of the component (the sensible zones don't cover the whole surface of the component). That is why we introduce the efficient section notion, expressed in $\mathrm{cm}^{2}$, that permits to indicate the component sensitivity to a given phenomenon.

This efficient section is expressed according to the rate of events accounted $N$ and the heavy ions flow corresponding to the normal flux at the component surface, in particles by $\mathrm{cm}^{2}$ and by second, that multiplies the irradiation time [13]:

$\sigma\left(\mathrm{cm}^{2}\right)=N$ (events) / (particle number $\left.\cdot \mathrm{cm}^{-2}\right)$.

To improve the analysis of the test results to the heavy ions, we can use a statistical data treatment. The component signature is therefore given by the couple $\left(L_{s}\right.$, $\sigma_{\text {sat }}$ ), where:

- $L_{s}$ is the threshold LET that corresponds to the minimal value dragging the apparition of at least an event on the component submitted to the heavy ions flux;

- $\sigma_{\text {sat }}$ is the saturation efficient section representing the maximal sensitive surface of the component.

The Weibull function used for the prediction of the efficient section led by heavy ions is given by $[9,14]$ (see Fig. 4)

$$
\begin{array}{ll}
\sigma(L)=\sigma_{0}\left[1-\exp \left(-\left(\frac{L-L_{0}}{W}\right)^{S}\right)\right], & L \geq L_{0} ; \\
\sigma(L)=0, & L<L_{0} .
\end{array}
$$

Where the four Bendel parameters are: $\sigma_{0}$ - asymptotic efficient section (by bit or by device); $L_{0}$ - threshold LET; $W$ - width parameter; $S$ - shape parameter.

\subsection{SEU lead by protons}

To the difference of the heavy ions, light ions such as protons have too weak LET $\left(<0.6 \mathrm{MeV} / \mathrm{mg} \cdot \mathrm{cm}^{2}\right)$ to be able to induce upsetting electrical effects in the components by a direct energy deposit [5].

The observed risks under a flux of protons involve receding nucleus obtained by nuclear interaction between the incident particle (proton) and atomic nucleus of the silicon. 
These secondaries relatively heavy and energizing can, for the same reason as the primary heavy ions of the cosmic radiance, have a sufficient LET to trigger upsets.

During the last ten years, several research teams were interested in the elaboration of prediction models of efficient sections of upset protons (EP). In our survey, we use the semi-empiric Bendel model. The equation giving the efficient section according to the energy takes the following shape $[13,15]$ (see Fig. 5)

$$
\begin{aligned}
& \sigma(E)=\left(\frac{24}{A}\right)^{14} \times \\
& \times\left\{1-\exp \left[-0.18 \cdot\left(\frac{18}{A}\right)^{1 / 4}\right](E-A)^{1 / 2}\right\}^{4}, E>A ; \\
& \sigma(E)=0, \text { elsewhere. }
\end{aligned}
$$

This functions gives good enough results for same devices, however with the components miniaturization another two parameters Bendel function is introduced [16]:

$$
\begin{aligned}
& \sigma(E)=\left(\frac{B}{A}\right)^{14} \times \\
& \times\left\{1-\exp \left[-0.18 \cdot\left(\frac{18}{A}\right)^{1 / 4}\right](E-A)^{1 / 2}\right\}^{4}, E>A ; \\
& \sigma(E)=0, \quad \text { elsewhere. }
\end{aligned}
$$

\section{Conclusion}

Our survey of the induced disruption in the VLSI circuits by the heavy ions and fast protons of the spatial environment has show-up major risks of physical failings apparition in electronic components embarked on board of space-crafts and satellite envolving on particulary exposed orbits.

These risks are associated to high ion and proton densities, whether they are localized in some magnetosphere zones (anomalies of the South Atlantic) or they result from natural unforseeable phenomena (solar eruptions).

In the perspective of a more complet orbital infrastructure, the economical stacke of the spatial programs bound to the development of the satellit constellations and the risk incured in human life making peremptory the electronic systems design that must be "freed" from the constraints associated to these phenomena.

\section{References}

1. L.D. Edmonds, Proton SEU cross sections derived from heavy-ion test data // IEEE Trans. on Nucl. SC. 47, N 5, October (2000).

2. R. Velazco, Predicting error rates provoked by SEUs in digital architectures: methods and tools, $5^{\text {th }}$
Microelectronics Reliability and Qualification Workshop (MRQW 2002).

3. C. Dyer, Radiation Effects on spacecraft \& aircraft, Space Department, QinetiQ, Cody Technology Park, Farnborough, Hampshire GU14 0LX, UK, Copyright QinetiQ Limited (2001).

4. T.A. Parnell, J.W. Watts, Jr. W. Armstrong, Radiation effects and protection for Moon and Mars missions.

5. Dj. Saadaoui, Etude de la fiabilité des Satellites en poste. Magister Université de Jijel Janvier (2004).

6. N. Merabtine, Modélisation de l'équation de Boltzmann par une conjecture sur l'équation de Landau Ginzburg. Thèse de Doctorat d'état ès Sciences, Université de Constantine Algérie, Octobre (2003).

7. N.J. Buchanan, D.M. Gingrich, Proton Induced Single-Event Upset Cross-Section of an SRAMBased FPGA. Center for Subatomic Research, University of Alberta, Edmonton, Alberta, Canada T6G 2N5, 2TRIUMF, Vancouver, British Columbia, Canada V6T 2A3 (2001).

8. D. Bosher, et al., Météorologie de L'environnement Spatial: Météo de L'espace. Rapport Final du Groupe D'évaluation des Besoins, Document CNES/DP/CM N 98-252.

9. D. Novak, Testing of electronics and development of detectors for radiation environments. Royal Institute of Technology, Stockholm (2001).

10. J.W. Howard, D.M. Hardage, Space Environments Interactions: Space Radiation and its Effects on Electronic Systems, National Aeronautics and Space Administration (NASA). Marshall Space Flight Center. MSFC, Alabama 35912, NASA TP-1999209373.

11. N. Buchanan, D.M. Guingritch, Proton induced Radiation Effects on a Xilins FPGA and Estimates of SEE in the ATLAS Environment, Center of Subatomic Research University of Alberta, Edmonton, AB, Canada, TRIUMF, Vancouver, BC, Canada, April 24, (2001).

12. M. Huhtinen, F. Faccio, Computational Method to Estimate Single Event Upset Rates in an Accelerator Environment, CERN, CH-1211 Geneva 23, Switzerland, Preprint submitted to Elsevier Preprint 11 October (1999).

13. N. Buchanan, D.M. Guingritch, Proton induced Radiation Effects on a Xilins FPGA and Estimates of SEE in the ATLAS Environment, Center of Subatomic Research University of Alberta, Edmonton, AB, Canada, TRIUMF, Vancouver, BC, Canada, April 24, (2001).

14. C. Laa, Comparison of CREME96 and Spenvis, Austrian Aerospace Ges.m.b.H (2002).

15. Radiation Effects Analysis Methods, Sira ElectroOptics Limited, South Hill Chislehurst, Kent, BR7 5EH, England, TN.245.2/DRAFT, August (2001). 